Boise State University

ScholarWorks

College of Social Sciences and Public Affairs

2014 Undergraduate Research and Scholarship

Presentations

Conference

4-21-2014

Prosocial Behavior and Posttraumatic Growth After Trauma

Rhiannon Trull

College of Social Sciences and Public Affairs, Boise State University 


\section{Prosocial Behavior and Posttrauamtic Growth After Trauma}

\section{Rhiannon Trull \\ Boise State University}

Faculty advisor: Dr. M. Rose Barlow

\section{Introduction}

- Previously thought to have been born from positive experiences, prosocial behavior may be one outcome after a traumatic event (Vollhardt, 2009). Prosocial behavior is acting in a manner that is meant to benefit others, such as volunteering, making donations, or expressing concern for the welfare of others. Trauma survivors may be motivated toward prosocial behavior in an attempt to alleviate negative affect related to personal suffering (Vollhardt, 2009). Frazier et al. (2012) found individuals who recently experienced an individual trauma engaged in more helping behaviors than those who had not experienced trauma.

- Triplett et al. (2012) noted individuals who indicated they were able to make sense of their traumatic experiences reported higher posttraumatic growth (PTG) and meaning in life. One of the proposed motivations for prosocia behavior following trauma is gaining meaning in life (Vollhardt, 2009). It is plausible that those who have increased tendencies toward prosocial behavior following trauma exhibit higher levels of PTG.

- Martin et al. (2013) suggested that as the number of types of trauma experienced increase, trauma-related symptomatology also increases. If the survivor of one type of trauma experiences additional traumatic events, it may impact the capability for positive change and lessen motivation for prosocial behavior.

\section{Hypotheses}

$\mathbf{H}_{\mathbf{1}}$ : Individuals who experienced an individual (personal) traumatic event will report higher levels of prosocial behavior than those who had not.

$\mathbf{H}_{2}$ : Individuals who have experienced trauma and who exhibit higher levels of prosocial behavior tendencies will report higher PTG than those who exhibit lower levels of prosocial behavior.

$\mathbf{H}_{3}$ : Individuals who experienced one type of trauma will report higher levels of PTG and higher tendencies toward prosocial behavior than those who have experienced more than one type of trauma.

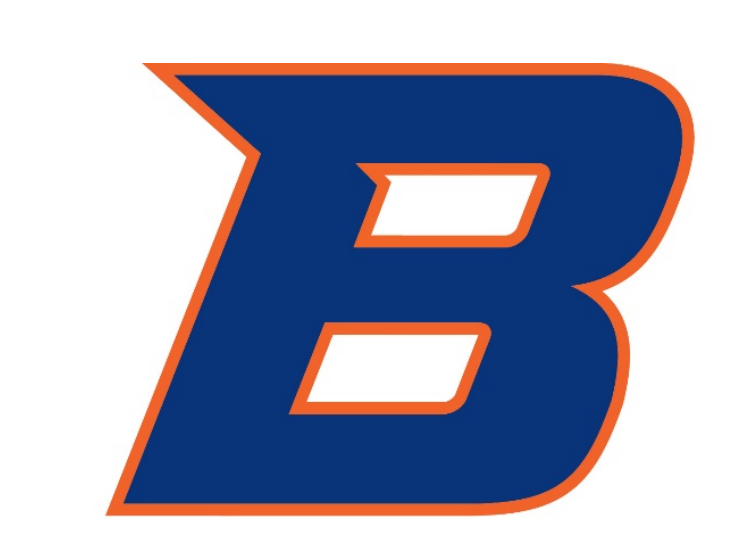

BOISE STATE UNIVERSITY

\section{Method}

Participants

A total of 312 undergraduate psychology students at Boise State University responded to an online survey. There were 125 males, 186 females, and one participant who did not indicate sex. The average age of participants was 20.14 years $(S D=4.15)$.

Measures

- Individual traumatic experiences were specified using the checklist from the Posttraumatic Stress Diagnostic Scale (Foa, Cashman, Jaycox, \& Perry, 1997).

- Prosocial behavior was measured by the Prosocial Tendencies Measure (PTM; Carlo \& Randall, 2002).

- PTG was measured by the Posttraumatic Growth InventoryShort Form (Cann et al., 2010).

\section{Results}

$\mathbf{H}_{1}$ : The first hypothesis was not supported. A one-way ANOVA revealed no significant difference in prosocial behavior between the trauma and no trauma groups $(F(1,279)=0.66, p=0.42)$. Participants who reported experiencing a traumatic event had a mean score of $75.56(S D=8.41)$ on the PTM and those who hed not experienced a traumatic event had a mean score of 74.56 ( $S D=$ 8.69).

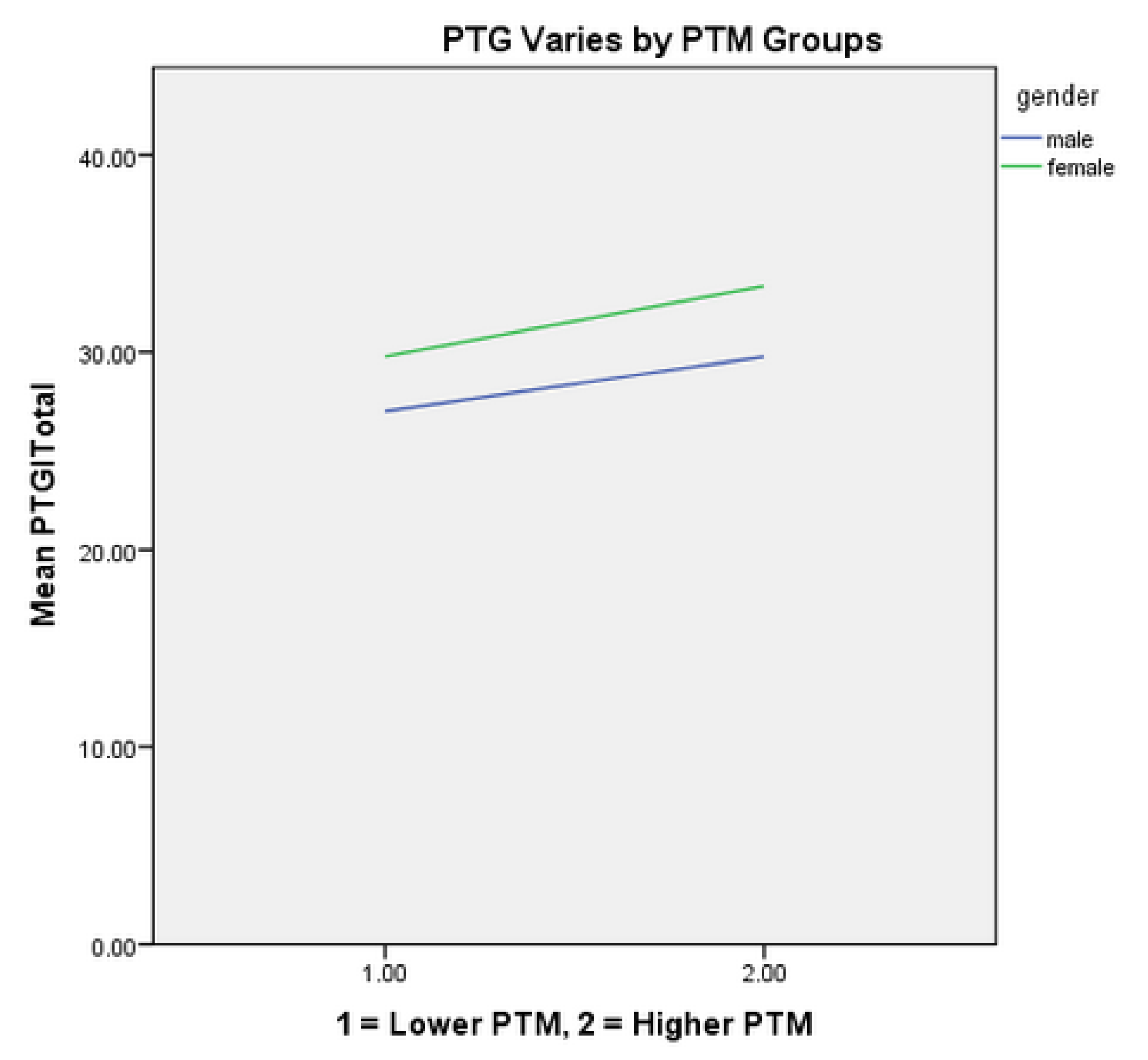

$\mathbf{H}_{2}$ : The second hypothesis was supported. A one-way ANOVA revealed a significant difference in PTG between those who reported lower prosocial behavior and those who reported higher prosocial behavior $(F(1,216)=7.35, p<0.01$, Cohen's $d=$ $0.367)$. Participants who reported higher prosocial behavior tendencies had higher PTG $(M=32.18, S D=9.95)$ than those who reported lower prosocial behavior tendencies $(M=28.48, S D=$ 10.19).

\section{Results cont.}

$\mathbf{H}_{3}$ : The third hypothesis was not supported. MANOVA results revealed that whether individuals experienced one or more than one type of trauma had no significant effect on the combined dependent variables of PTG and prosocial behavior tendencies [Wilks' $\Lambda=0.99, F(2,215)=1.15, p=$ $\left.0.32, \eta^{2}=0.011\right]$. Participants who had experienced more than one type of trauma had slightly higher tendencies toward prosocial behavior $(M=76.21, S D=8.40)$ than those who experienced only one trauma type $(M=74.57, S D=$ 9.38) but results were not significant. The mean PTG score was $29.28(S D=10.64)$ for those who experienced one type of trauma, and those who experienced more than one type of trauma had a mean PTG score of $30.84(S D=9.99)$.

\section{Discussion}

- Propensity toward prosocial behavior does not seem to differ based whether an individual has experienced a traumatic event. Individuals who experienced at least one traumatic event and who reported higher tendencies toward prosocial behavior reported higher levels of PTG than those who reported lower tendencies. These findings suggest that individuals who have experienced a traumatic event followed by positive personal change may feel better situated to assist others.

- The lack of significant difference between those who experienced one or more than one type of traumatic event in prosocial behavior and PTG may be attributed to the nature of the sample. Opportunities for personal growth while in college may help to mitigate the negative effects of having experienced more than one type of trauma. Anderson and Lopez-Baez (2011) effectively measured personal growth using the PTGI in undergraduate students who did not experience a traumatic event and participants attributed their growth to educational activities which offered various tools and resources for facilitating personal growth

- Taken together, the results of this study suggest that prosocial behavior may have a stronger association to PTG following an individual trauma than with traumatic experiences.

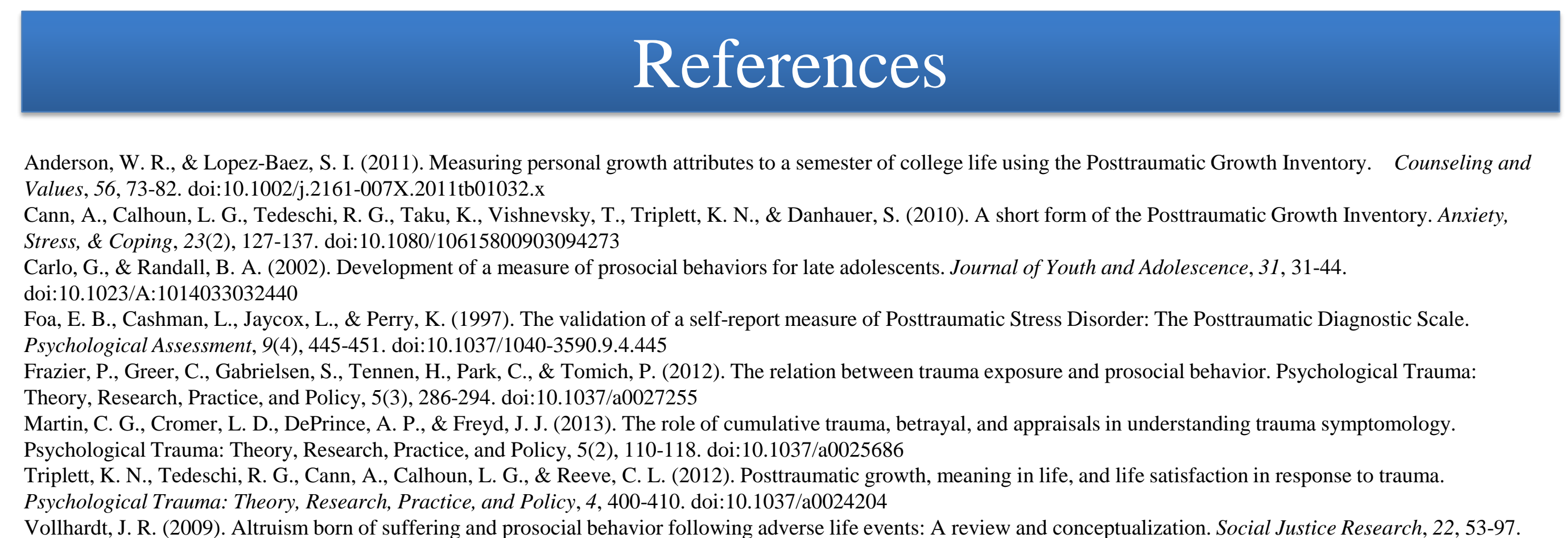

